IMAFF 93/11

LPTHE-PAR 93/21

April 1993

\title{
Free Fermionic Elliptic Reflection Matrices and Quantum Group Invariance
}

\author{
R. Cuerno \\ Instituto de Matemáticas y Física Fundamental, CSIC \\ Serrano 123, E-28006 Madrid, SPAIN \\ A. González-Ruiz* \\ L.P.T.H.E. \\ Tour 16, 1er étage, Université Paris VI \\ 4 Place Jussieu, 75252 Paris cedex 05, FRANCE
}

\begin{abstract}
Diagonal solutions for the reflection matrices associated to the elliptic $R$ matrix of the eight vertex free fermion model are presented. They lead through the second derivative of the open chain transfer matrix to an XY hamiltonian in a magnetic field which is invariant under a quantum deformed Clifford-Hopf algebra.
\end{abstract}

*Permanent address: Departamento de Física Teórica, Universidad Complutense, 28040 Madrid, SPAIN 
A subject which is focusing great attention in Mathematical Physics is to find a quantum group-like structure in which Baxter's zero field 8 vertex matrix [1] could act as an intertwiner (for a recent attempt see [2]). Recently a solution has been found for the related case of the free-fermionic elliptic eight vertex matrix introduced in [3, 4]: it appears as an intertwiner for the affinization of a quantum Hopf deformation of the Clifford algebra in two dimensions, $C \widehat{H_{q}}(2)$ [5]. In the trigonometric limit this free-fermionic $R$ matrix becomes the intertwiner of the quantum algebra $\mathcal{U}_{\hat{q}}(g l \widehat{(1,1)})$ [6], , $]$, or can also be seen as the intertwiner for non classical nilpotent irreducible representations of $\mathcal{U}_{q}(\widehat{s l(2)})$ for $q^{4}=1$ and $\hat{q}=\lambda$, with $\lambda^{2}$ the eigenvalue of the casimir $K^{2}$ [8, 9]. An integrable open chain hamiltonian can be constructed for this trigonometric matrix [10, 11] which is invariant under the $\mathcal{U}_{q}(s l(2))$ algebra in nilpotent irreps [12] (also, see [13] for the analogous $q^{3}=1$ case).

In this letter we consider the open chain hamiltonian associated with the elliptic free fermionic matrix $R(u)$ (11) [3, 4], $u$ being the complex spectral parameter living on a torus. Elliptic $K$ matrices will appear as solutions to the associated reflection equations [10, 11], and analogously to the trigonometric case the associated open chain hamiltonian will be invariant under the non-affine subalgebra of the bigger structure for which the $R(u)$ matrix acts as an intertwiner. First we will introduce the relevant material on $C \widehat{H_{q}}(2)$ and $\mathrm{CH}_{q}(2)$, and after working out the trigonometric (nilpotent) case to fix notations and procedure we will consider the elliptic one: by the use of the $K$ matrices an integrable open chain hamiltonian is constructed which is invariant under the quantum algebra $\mathrm{CH}_{q}(2)$. It is no other but an XY model in a magnetic field. See 14 for a different approach to its quantum group invariance. In all these free fermionic models (both in the trigonometric and in the elliptic cases) an analogous result is obtained: $\operatorname{Tr} K_{+}(0)=0$. It forces us to define the hamiltonian of the associated spin model through the second derivative of Sklyanin's [11] open chain transfer matrix; only nearest-neighbour spins turn out to be coupled in contrast to what could be expected [15. Some final comments are also included.

The most general solution for the elliptic free fermionic $8 \mathrm{~V} R$ matrix is, in the parametrization of ref. [4]:

$$
\begin{aligned}
& R_{00}^{00}=1-e(u) e\left(\psi_{1}\right) e\left(\psi_{2}\right) \\
& R_{11}^{11}=e(u)-e\left(\psi_{1}\right) e\left(\psi_{2}\right) \\
& R_{01}^{01}=e\left(\psi_{2}\right)-e(u) e\left(\psi_{1}\right)
\end{aligned}
$$




$$
\begin{aligned}
& R_{10}^{10}=e\left(\psi_{1}\right)-e(u) e\left(\psi_{2}\right) \\
& R_{01}^{10}=R_{10}^{01}=\left(e\left(\psi_{1}\right) \operatorname{sn}\left(\psi_{1}\right)\right)^{1 / 2}\left(e\left(\psi_{2}\right) \operatorname{sn}\left(\psi_{2}\right)\right)^{1 / 2}(1-e(u)) / \operatorname{sn}(u / 2) \\
& R_{00}^{11}=R_{11}^{00}=-\mathrm{i} k\left(e\left(\psi_{1}\right) \operatorname{sn}\left(\psi_{1}\right)\right)^{1 / 2}\left(e\left(\psi_{2}\right) \operatorname{sn}\left(\psi_{2}\right)\right)^{1 / 2}(1+e(u)) \operatorname{sn}(u / 2)
\end{aligned}
$$

with $e(u)$ the elliptic exponential:

$$
e(u)=\operatorname{cn}(u)+\mathrm{i} \operatorname{sn}(u)
$$

$k$ the elliptic modulus and $\operatorname{cn}(u), \operatorname{sn}(u)$ the Jacobi elliptic functions of modulus $k$. In the sequel we will set

$$
\psi_{1}=\psi_{2} \equiv \psi
$$

The quantum deformed Clifford-Hopf algebra of dimension $D=2, C H_{q}(2)$ [5], is generated by $\Gamma_{\mu}(\mu=x, y), \Gamma_{3}$ and the central elements $E_{\mu}(\mu=x, y)$ satisfying the following relations:

$$
\begin{aligned}
& \Gamma_{\mu}^{2}=\frac{q^{E_{\mu}}-q^{-E_{\mu}}}{q-q^{-1}}, \quad \Gamma_{3}^{2}=\mathbf{1} \\
& \left\{\Gamma_{x}, \Gamma_{y}\right\}=0, \quad\left\{\Gamma_{\mu}, \Gamma_{3}\right\}=0 \\
& {\left[E_{\mu}, \Gamma_{\nu}\right]=\left[E_{\mu}, \Gamma_{3}\right]=\left[E_{\mu}, E_{\nu}\right]=0 \quad \forall \mu, \nu}
\end{aligned}
$$

It is a Hopf algebra with the following comultiplication $\Delta$, antipode $S$ and counit $\epsilon$ :

$$
\begin{array}{lll}
\Delta\left(E_{\mu}\right)=E_{\mu} \otimes \mathbf{1}+\mathbf{1} \otimes E_{\mu}, & S\left(E_{\mu}\right)=-E_{\mu}, & \epsilon\left(E_{\mu}\right)=0 \\
\Delta\left(\Gamma_{\mu}\right)=\Gamma_{\mu} \otimes q^{-E_{\mu} / 2}+q^{E_{\mu} / 2} \Gamma_{3} \otimes \Gamma_{\mu}, & S\left(\Gamma_{\mu}\right)=\Gamma_{\mu} \Gamma_{3}, & \epsilon\left(\Gamma_{\mu}\right)=0 \\
\Delta\left(\Gamma_{3}\right)=\Gamma_{3} \otimes \Gamma_{3}, & S\left(\Gamma_{3}\right)=\Gamma_{3}, & \epsilon\left(\Gamma_{3}\right)=1
\end{array}
$$

A two dimensional representation is labelled by two complex parameters $\xi=\left(\lambda_{x}, \lambda_{y}\right)$ and is given by

$$
\begin{aligned}
& \pi_{\xi}\left(\Gamma_{x}\right)=\left(\frac{\lambda_{x}-\lambda_{x}^{-1}}{q-q^{-1}}\right)^{1 / 2} \sigma_{x}, \pi_{\xi}\left(\Gamma_{y}\right)=\left(\frac{\lambda_{y}-\lambda_{y}^{-1}}{q-q^{-1}}\right)^{1 / 2} \sigma_{y} \\
& \pi_{\xi}\left(\Gamma_{3}\right)=\sigma_{z}, \pi_{\xi}\left(q^{E_{x}}\right)=\lambda_{x}, \pi_{\xi}\left(q^{E_{y}}\right)=\lambda_{y}
\end{aligned}
$$

where $\left\{\sigma_{j}\right\}_{j=x, y, z}$ are Pauli matrices. The (sort of) affine extension of this algebra, $C \widehat{H_{q}}(2)$, is generated by $E_{\mu}^{(i)}, \Gamma_{\mu}^{(i)}, \quad(i=0,1)$ and $\Gamma_{3}$ satisfying (国) and (畐) for each value of $i$. Now a two dimensional irrep $\pi_{\xi}$ of $C \widehat{H_{q}}(2)$ is labelled by three complex parameters $\xi=\left(z, \lambda_{x}, \lambda_{y}\right) \in C_{\times}^{3}$ which fulfill the following constraint:

$$
\frac{2\left(\lambda_{x}-\lambda_{y}\right)}{\left(1-\lambda_{x}^{2}\right)^{1 / 2}\left(1-\lambda_{y}^{2}\right)^{1 / 2}\left(z^{2}-z^{-2}\right)}=k
$$


See more details in [5]. There (11) is shown to be the intertwiner between two irreps which satisfy (6) for the same value of $k$, with:

$$
\begin{aligned}
z^{2} & =\operatorname{cn}(\varphi)+\mathrm{i} \operatorname{sn}(\varphi) \\
\lambda_{x} & =\tanh x, \lambda_{y}=\tanh y \\
\tanh \left(\frac{x+y}{2}\right) & =\operatorname{cn}(\psi)+\mathrm{i} \operatorname{sn}(\psi) \\
u & =\varphi_{1}-\varphi_{2}
\end{aligned}
$$

i.e. one has

$$
\check{R}_{\xi_{1} \xi_{2}} \Delta_{\xi_{1} \xi_{2}}(a)=\Delta_{\xi_{2} \xi_{1}}(a) \check{R}_{\xi_{1} \xi_{2}} \quad \forall a \in C \widehat{H_{q}}(2)
$$

for $\check{R}(u) \equiv \mathcal{P} R(u), \mathcal{P}$ being the permutation matrix.

Consider now the trigonometric limit of the elliptic matrix (11). It can be written as

$$
R(\theta)=\frac{1}{\lambda-\lambda^{-1}}\left(\begin{array}{llll}
e^{\theta} \lambda-e^{-\theta} \lambda^{-1} & & \\
& e^{\theta}-e^{-\theta} & \lambda-\lambda^{-1} & \\
& \lambda-\lambda^{-1} & e^{\theta}-e^{-\theta} & \\
& & & e^{-\theta} \lambda-e^{\theta} \lambda^{-1}
\end{array}\right)
$$

with $\lambda \equiv e^{\mathrm{i} \psi}, \theta \equiv \frac{\mathrm{i}}{2} u$. All Skylanin's requirements on symmetries (see [11 and (18)), are fulfilled with anisotropy fixed to $\eta=\pi / 2$. The reflection-factorization equations for diagonal $K_{ \pm}(\theta)$ matrices, which are the ones considered in this letter, are:

$$
\begin{aligned}
& R_{12}\left(\theta_{1}-\theta_{2}\right) \stackrel{1}{K}{ }_{-}\left(\theta_{1}\right) R_{12}\left(\theta_{1}+\theta_{2}\right) \stackrel{2}{K}-\left(\theta_{2}\right)= \\
& =\stackrel{2}{K}-\left(\theta_{2}\right) R_{12}\left(\theta_{1}+\theta_{2}\right) \stackrel{1}{K}-\left(\theta_{1}\right) R_{12}\left(\theta_{1}-\theta_{2}\right) \\
& R_{12}\left(-\theta_{1}+\theta_{2}\right) \stackrel{1}{K}+\left(\theta_{1}\right) R_{12}\left(-\theta_{1}-\theta_{2}-2 \eta\right) \stackrel{2}{K}+\left(\theta_{2}\right)= \\
& =\stackrel{2}{K}\left(\theta_{2}\right) R_{12}\left(-\theta_{1}-\theta_{2}-2 \eta\right) \stackrel{1}{K}\left(\theta_{1}\right) R_{12}\left(-\theta_{1}+\theta_{2}\right)
\end{aligned}
$$

Cherednik's solutions to these in the case of the $6 \mathrm{~V}$ model are also valid for (8) with the mentioned requirement that $\eta=\pi / 2$. This implies in particular that

$$
\operatorname{Tr} K_{+}(0)=0
$$

which as anticipated makes it troublesome to define the open chain hamiltonian through the standard formula [11]:

$$
H=\sum_{j=1}^{N-1} H_{j, j+1}+\frac{1}{2} \stackrel{1}{K}^{\prime}-(0)+\frac{\operatorname{Tr}_{0} \stackrel{0}{K}_{+}(0) H_{N 0}}{\operatorname{Tr} K_{+}(0)}
$$




$$
\left.H_{j, j+1} \equiv \mathcal{P}_{j, j+1} \frac{d R(\theta)}{d \theta}\right|_{\theta=0}
$$

However, using (11) and the fact that

$$
\operatorname{Tr}_{0} \stackrel{0}{K}+(0) H_{N 0}=A \mathbf{1}
$$

where $A$ is a constant, we can see that there still exists a well defined open chain hamiltonian defining it as [13]:

$$
\begin{aligned}
H \equiv & \frac{t^{\prime \prime}(0)}{4(T+2 A)}= \\
= & \sum_{j=1}^{N-1} H_{j, j+1}+\frac{1}{2} \stackrel{{ }^{\prime}}{K_{-}}(0)+\frac{1}{2(T+2 A)} \times \\
& \left\{\operatorname{Tr}_{0}\left(\stackrel{0}{K}+(0) G_{N 0}\right)+2 \operatorname{Tr}_{0}\left(\stackrel{0}{K}_{+}^{\prime}(0) H_{N 0}\right)+\operatorname{Tr}_{0}\left(\stackrel{0}{K_{+}}(0) H_{N 0}^{2}\right)\right\}
\end{aligned}
$$

where $t(u)$ is Sklyanin's open chain transfer matrix, and:

$$
\begin{aligned}
T & \equiv \operatorname{Tr} K_{+}^{\prime}(0) \\
G_{j, j+1} & \left.\equiv \mathcal{P}_{j, j+1} \frac{d^{2} R_{j, j+1}(\theta)}{d \theta^{2}}\right|_{\theta=0}
\end{aligned}
$$

For the case of (8) one gets the following hamiltonians:

$$
H=\frac{1}{2} \sum_{j=1}^{N-1}\left\{\sigma_{j}^{x} \sigma_{j+1}^{x}+\sigma_{j}^{y} \sigma_{j+1}^{y}+\frac{\lambda+\lambda^{-1}}{2}\left(\sigma_{j}^{z}+\sigma_{j+1}^{z}\right)\right\} \pm \frac{1}{4}\left(\lambda-\lambda^{-1}\right)\left(\sigma_{1}^{z}-\sigma_{N}^{z}\right)
$$

where we have taken the standard limits $\alpha_{ \pm} \rightarrow \pm \infty$ in the arbitrary parameters appearing in the $K_{ \pm}(\theta)$ matrices [16]. This leaves us with an XX model in a magnetic field which is invariant under $\mathcal{U}_{q}(s l(2))$ transformations in nilpotent irrep $\lambda$ for $q^{4}=1$, i.e.

$$
\left[H, \Delta^{(N)}(g)\right]=0
$$

with $g$ any generator of $\mathcal{U}_{q}(s l(2))$ in the nilpotent irrep $\lambda$ for $q^{4}=1$ (see [17 for a treatment of general nilpotent closed chain spin models) and $\Delta^{(N)}$ the comultiplication applied $N$ times (for an $N$ site chain).

We turn now our attention to the elliptic $R$ matrix (1). Taking $\psi_{1}=\psi_{2}=\psi$ it can be seen to have the following set of symmetries:

$$
R(0)=\left(1-e(\psi)^{2}\right) \mathcal{P}
$$




$$
\begin{aligned}
\mathcal{P} R(u) \mathcal{P} & =R(u)=R^{t_{12}}(u) \\
R(u) R(-u) & =\rho(u) \mathbf{1} \\
R^{t_{1}}(u) R^{t_{1}}(-u+4 K) & =\tilde{\rho}(u) \mathbf{1}
\end{aligned}
$$

with $\rho(u), \tilde{\rho}(u)$ some unimportant scalar functions and $K$ the complete elliptic integral of the first kind of modulus $k$. The most general diagonal solutions to the corresponding reflection equations (9), (10) are:

$$
\begin{aligned}
& K_{-}(u)=\left(\begin{array}{ll}
\operatorname{cn}\left(\frac{u}{2}\right) \operatorname{dn}\left(\frac{u}{2}\right) \pm \mathrm{i} k^{\prime} \operatorname{sn}\left(\frac{u}{2}\right) & \\
\operatorname{cn}\left(\frac{u}{2}\right) \operatorname{dn}\left(\frac{u}{2}\right) \mp \mathrm{i} k^{\prime} \operatorname{sn}\left(\frac{u}{2}\right)
\end{array}\right) \\
& K_{+}(u)=\left(\begin{array}{ll}
k^{\prime 2 \frac{\operatorname{sn}\left(\frac{u}{2}\right)}{\operatorname{dn}^{2}\left(\frac{u}{2}\right)} \pm \mathrm{i} k^{\prime} \frac{\operatorname{cn}\left(\frac{u}{2}\right)}{\operatorname{dn}\left(\frac{u}{2}\right)}} & \\
& k^{\prime 2} \frac{\operatorname{sn}\left(\frac{u}{2}\right)}{\operatorname{dn}^{2}\left(\frac{u}{2}\right)} \mp \mathrm{i} k^{\prime} \frac{\operatorname{cn}\left(\frac{u}{2}\right)}{\operatorname{dn}\left(\frac{u}{2}\right)}
\end{array}\right)
\end{aligned}
$$

where $k^{\prime}$ is the complementary elliptic modulus $k^{2}+k^{\prime 2}=1$, and we have used that $K_{+}(u)=K_{-}(-u+2 K)$ solves (10). Note that there is no dependence on arbitrary parameters. This seems to be the general case for elliptic $R$ matrices; a dependence on an arbitrary parameter is expected, however, for non diagonal $K$ matrices [18]. Moreover the trigonometric limit of these matrices give us the ones that yield the quantum group invariant hamiltonian (16). Note that we again have (11) and (14) even at the elliptic level. So, whereas the symmetry properties (18) enable us to construct the transfer matrix of an integrable open chain model à la Sklyanin [11, we again have to resort to (15) to construct the corresponding spin hamiltonian. In this case the solutions are given by

$$
H=\frac{1}{2} \sum_{j=1}^{N}\left\{(1+\Gamma) \sigma_{j}^{x} \sigma_{j+1}^{x}+(1-\Gamma) \sigma_{j}^{y} \sigma_{j+1}^{y}+h\left(\sigma_{j}^{z}+\sigma_{j+1}^{z}\right)\right\} \pm \frac{\mathrm{i} k^{\prime} \operatorname{sn}(\psi)}{2}\left(\sigma_{1}^{z}-\sigma_{N}^{z}\right)
$$

where $h=\operatorname{cn}(\psi)$, and $\Gamma=k \operatorname{sn}(\psi)$. (We have dropped in (19) a term proportional to the identity operator). (19) gives (16) in the trigonometric limit. The hamiltonians in (19) are invariant under $\mathrm{CH}_{q}(2)$ transformations in representations labelled by

$$
\lambda_{x}=\frac{\operatorname{cn}(\psi) \pm \mathrm{i} k^{\prime} \operatorname{sn}(\psi)}{1+k \operatorname{sn}(\psi)}, \quad \lambda_{y}=\frac{\operatorname{cn}(\psi) \pm \mathrm{i} k^{\prime} \operatorname{sn}(\psi)}{1-k \operatorname{sn}(\psi)}
$$

i.e. they fulfill commutation relations like (17) for $g$ any generator of $C H_{q}(2)$ in the above representations.

We have shown that the $K$ matrices associated with the elliptic free fermion $R$ matrix lead to an open chain hamiltonian which is explicitly invariant under a quantum algebra. 
Investigating a similar phenomenon would be interesting in the case of the zero field $8 \mathrm{~V}$ model. Also it would be interesting to examine the implications of non-diagonal solutions for the $K$ matrices in both cases. We have found here the appearance of the conditions $\operatorname{Tr} K_{+}(0)=0$ and $\operatorname{Tr}\left(\stackrel{0}{K}{ }_{+}(0) H_{N 0}\right)=A 1$ also at the elliptic level. In the trigonometric case they can be interpreted in terms of quantum group representation theoretical terms [19, 20]. It would be interesting to find the explanation for the elliptic occurrence of the same conditions. In this connection it would also be of interest to find some "gauge transformation" which would gauge out the $u$ dependence from the elliptic $K$ matrices [21], while taking $R(u)$ (11) to a baxterized form [22]. Finally the Algebraic Bethe Anstaz [11] for the elliptic open chain described here would also be of interest, as well as a detailed study of the algebraic "contraction" involved in the trigonometric limit $C H_{q}(2) \rightarrow \mathcal{U}_{q}(g l(1,1))$. We hope to report on these matters in future publications.

\section{Acknowledgments}

RC is pleased to thank A. Berkovich, C. Gómez, E. López and G. Sierra for discussions and encouragement. AGR thanks the CNRS Univ. Paris VI for the kind hospitality extended to him and H.J. de Vega for valuable discussions. The research of both authors is supported by the Spanish Ministry of Education and Science through predoctoral fellowships PN89-11798388 (RC) and AP90-02620085 (AGR). 


\section{References}

[1] R.J.Baxter, Exactly solved models in statistical mechanics, Academic Press, 1982.

[2] D.B.Uglov, "The Lie algebra of the $s l(2, \mathcal{C})$-valued automorphic finctions on a torus", and "The quantum bialgebra associated with the eight-vertex R-matrix", SUNY preprints, February 1993.

[3] B.U.Felderhof, Physica 66 (1973) 279.

[4] V.V.Bazhanov and Yu.G.Stroganov, Teor. Mat. Fiz. 62 (1985) 37.

[5] R.Cuerno, C.Gómez, E.López and G.Sierra, "The Hidden Quantum Group of the 8vertex Free Fermion Model: q-Clifford Algebras", preprint IMAFF-2/93, to appear in Phys. Lett. B.

[6] L.H.Kauffman and H.Saleur, Commun. Math. Phys. 141 (1991) 293.

[7] L.Rozansky and H.Saleur, Nucl. Phys. B376 (1992) 461.

[8] J.Murakami, Int. Jour. Mod. Phys. A7, Suppl. 1B (1992) 765.

[9] M.Ruiz-Altaba, Phys. Lett. B279 (1992) 326.

[10] I.V.Cherednik, Teor. Mat. Fiz. 61 (1984) 35.

[11] E.K.Sklyanin, Jour. Phys. A:Math. Gen. 21 (1988) 2375.

[12] D.Arnaudon and V.Rittenberg, "Quantum Chains with $\mathcal{U}_{q}(\operatorname{sl}(2))$ Symmetry and Unrestricted Representations", preprint CERN-TH.6786/93, January 1993.

[13] R.Cuerno, C.Gómez and G.Sierra, Jour. Geom. Phys. 11 (1993).

[14] H.Hinrichsen and V.Rittenberg, Phys. Lett. B275 (1992) 350, and preprint BONN HE-93-02.

[15] M.Lüscher, Nucl. Phys. B117 (1976) 475.

[16] C.Destri and H.J.de Vega, Nucl. Phys. B374 (1992) 692.

[17] A.Berkovich, C.Gómez and G.Sierra, "Spin-Anisotropy Commensurable Chains: Quantum Group Symmetries and N=2 SUSY", preprint IMAFF-93-1, January 1993. 
[18] H.J.de Vega and A.González-Ruiz, in preparation.

[19] L.Mezincescu and R.I.Nepomechie, Int. Jour. Mod. Phys. A6 (1991) 5231 and addendum Int. Jour. Mod. Phys. A7 (1992) 5657.

[20] R.Cuerno, in preparation.

[21] L.Mezincescu and R.I.Nepomechie, Jour. Phys. A:Math. Gen. 24 (1991) L17.

[22] V.F.R.Jones, Int. Jour. Mod. Phys. A6 (1991) 2035. 